\title{
Comparison of resting-state EEG between adults with Down syndrome and typically developing controls
}

\author{
Sarah Hamburg ${ }^{1 *}$ (D), Daniel Bush ${ }^{2,3}$, Andre Strydom ${ }^{1,4}$ and Carla M. Startin ${ }^{1,5}$
}

\begin{abstract}
Background: Down syndrome (DS) is the most common genetic cause of intellectual disability (ID) worldwide. Understanding electrophysiological characteristics associated with DS provides potential mechanistic insights into ID, helping inform biomarkers and targets for intervention. Currently, electrophysiological characteristics associated with DS remain unclear due to methodological differences between studies and inadequate controls for cognitive decline as a potential cofounder.

Methods: Eyes-closed resting-state EEG measures (specifically delta, theta, alpha, and beta absolute and relative powers, and alpha peak amplitude, frequency and frequency variance) in occipital and frontal regions were compared between adults with DS (with no diagnosis of dementia or evidence of cognitive decline) and typically developing (TD) matched controls ( $n=25$ per group).

Results: We report an overall 'slower' EEG spectrum, characterised by higher delta and theta power, and lower alpha and beta power, for both regions in people with DS. Alpha activity in particular showed strong group differences, including lower power, lower peak amplitude and greater peak frequency variance in people with DS.

Conclusions: Such EEG 'slowing' has previously been associated with cognitive decline in both DS and TD populations. These findings indicate the potential existence of a universal EEG signature of cognitive impairment, regardless of origin (neurodevelopmental or neurodegenerative), warranting further exploration.
\end{abstract}

Keywords: Down syndrome, Trisomy 21, EEG, Resting state, Alpha peak

\section{Introduction}

Down syndrome (DS) is caused by an extra copy of chromosome 21 and is the most common genetic cause of intellectual disability (ID) worldwide, affecting 1 in 800 births [1]. Due to a 'triple dose' of genes on this chromosome, almost all individuals with DS have an ID (clinically defined as an IQ less than 70 and impairments in everyday adaptive abilities), in addition to an ultrahigh risk of developing Alzheimer's disease (AD) with a lifetime prevalence of $90 \%$ [2]. Understanding brain

\footnotetext{
* Correspondence: s.hamburg@ucl.ac.uk

${ }^{1}$ The London Down Syndrome Consortium (LonDownS), London, UK

Full list of author information is available at the end of the article
}

function in people with DS is important for elucidating these characteristics, and electrophysiological measures in particular allow us to examine potential mechanisms related to function. Understanding electrophysiological characteristics associated with DS may therefore provide mechanistic insights into both cognitive ability and decline.

Resting-state electroencephalography (EEG) paradigms provide a general measure of brain activity (i.e. activity that is not associated with any particular sensory modality). As resting-state paradigms are passive, they are inherently free from the need for participants to understand and retain task instructions (e.g. pressing a

C C The Author(s). 2021 Open Access This article is licensed under a Creative Commons Attribution 4.0 International License, which permits use, sharing, adaptation, distribution and reproduction in any medium or format, as long as you give appropriate credit to the original author(s) and the source, provide a link to the Creative Commons licence, and indicate if changes were made. The images or other third party material in this article are included in the article's Creative Commons licence, unless indicated otherwise in a credit line to the material. If material is not included in the article's Creative Commons licence and your intended use is not permitted by statutory regulation or exceeds the permitted use, you will need to obtain permission directly from the copyright holder. To view a copy of this licence, visit http://creativecommons.org/licenses/by/4.0/ The Creative Commons Public Domain Dedication waiver (http://creativecommons.org/publicdomain/zero/1.0/) applies to the data made available in this article, unless otherwise stated in a credit line to the data. 
button in response to a target), thus reducing any confounding influences of individual differences in ID level and motor skills [3]. Such paradigms are therefore suitable for use with the majority of individuals with DS.

EEG recordings reveal oscillatory brain activity. The organisation of this activity represents the means by which neuronal networks dynamically communicate and interact [4]. For example, networks of inhibitory interneurons generate rhythmic inhibitory post-synaptic potentials (IPSPs). These rhythmic IPSPs provide windows of alternating reduced and enhanced excitability, offering a temporal framework for the 'chunking' of neuronal activity. This 'chunking' enables the effective communication of local information to distributed regions. It is posited that these mechanisms enable the brain to integrate a large number of distributed local processes into global states [4]. Such brain rhythms are characterised by distinct frequency bands, associated with differing underlying mechanisms of generation and brain functions; typically, delta $(<4 \mathrm{~Hz})$, theta $(4-8$ $\mathrm{Hz})$, alpha $(8-13 \mathrm{~Hz})$, beta $(13-30 \mathrm{~Hz})$ and gamma (> $30 \mathrm{~Hz}$ ). It is of note, however, that brain rhythms may operate across a wider frequency range than these discrete categories, with substantial intersubject variability [5].

Using EEG to identify individual and group differences in oscillatory brain activity has been of interest to researchers since EEG was invented in the 1920s. Power in both slow (delta and theta) and fast (beta and gamma) EEG bands has generally been reported as greater in participants with DS compared to typically developing (TD) controls [6-14]. This pattern is well established for slower frequencies, though inconsistencies in the literature exist for faster frequencies, with Babiloni et al. [12, 13] reporting less power in beta and gamma bands in individuals with DS compared to TD controls.

As alpha waves can be visually identified in EEG recordings, they have been of particular interest to DS researchers from the earliest EEG studies [15]. Alpha activity is commonly associated with both IQ and memory performance in the TD population [16-18], in addition to between people with DS [19]. Differences in alpha activity between people with DS and TD controls are commonly reported, though specific findings are inconsistent. Whilst the majority of studies have reported less alpha power in DS $[8,11,12]$, others have found significantly more alpha power in DS [9] or no difference between DS and TD controls [10]. For alpha peak frequency (the frequency at which peak amplitude occurs within this band), many studies have found people with DS have a significantly slower peak frequency $[6,7,11,14,20]$, though others reported no significant difference $[10,13]$.
These inconsistencies in findings, for alpha power in particular, are in part likely due to methodological differences between EEG studies. Differences in study characteristics are common, including frequency band classification, power measure (i.e. relative or absolute), scalp regions examined, participant age and whether any participants show signs of cognitive decline. For example, Politoff et al. [10] reported no significant differences between people with DS and TD controls for relative power (where power is calculated relative to the total EEG power for each participant) but found differences for absolute power. There is therefore value in examining both type of power measure. Previous research also shows topographical differences are important to consider-differences in alpha activity between people with DS and TD controls appear most apparent in posterior regions $[8,11]$ and may differ between occipital and parietal electrode derivations [6], whilst delta differences may be most apparent in frontal and centroanterior regions $[8,11,12]$, theta differences in centroposterior regions $[8,11]$, and beta differences in parietotemporal regions $[8,11]$.

Given the previous discrepancies in the literature regarding differences in EEG measures between people with DS and TD controls, and the potential contribution of methodological variations to these discrepancies, it is important to conduct research accounting for these variations to understand differences in EEG activity between people with DS and TD controls. We therefore compared EEG activity between adults with DS and TD controls using commonly used frequency band classifications, and both absolute and relative power, in addition to including two scalp regions (occipital and frontal). To reduce a potential confounding effect of cognitive decline, we used a sample of adults with DS with no noticeable cognitive decline. Understanding these differences in activity between adults with DS and TD controls will not only provide mechanistic insights into cognitive ability but also help elucidate the significance of studies examining individual differences between people with DS. In turn, this may help inform biomarker and drug target research.

Based on previous findings, it was hypothesised that individuals with DS would have less alpha power (8$13 \mathrm{~Hz})$ but more power in delta $(0.5-4 \mathrm{~Hz})$, theta $(4-8$ $\mathrm{Hz})$ and beta $(13-30 \mathrm{~Hz})$ bands compared to TD controls. Results were not expected to significantly differ between absolute and relative power measures, or between occipital and frontal electrode montages. Gamma activity was not investigated as it shares a similar frequency to muscle artefacts, which are common in electrophysiological recordings in people with DS due to lower compliance with the instruction to remain still. 


\section{Methods}

\section{Participants}

Participants with DS were recruited from an existing pool of UK adults with DS who had participated in an initial cognitive assessment [3]. All participants had genetically confirmed trisomy 21 and were aged 16 and over. Participants with an acute physical or mental health condition were excluded, as were participants with a clinical diagnosis of dementia or the presence of cognitive decline associated with dementia. The presence of cognitive decline was determined by the Cambridge Examination of Mental Disorders of Older People with Down Syndrome and Others with Intellectual Disabilities (CAMDEX-DS [21]), which is considered a valid and reliable tool for assessing cognitive decline in adults with DS [21]. It is an informant-based questionnaire which enquires about decline (with respect to an individuals' best level of functioning) within the following domains: everyday skills, memory, orientation, general mental functioning, language, perception, praxis, executive functions, personality, behaviour and self-care. Any change in any one of these domains was scored as presence of decline. All participants were required to show no decline on this questionnaire to be included in the study. The resting-state EEG recordings from all participants with DS selected for this study has also previously been used in a separate investigation into differences between individuals with DS [19].

TD control group participants were selected from the Multimodal Resource for Studying Information Processing in the Developing Brain (MIPDB) [22]. The MIPDB is a large open source dataset provided by the Child Mind Institute. This dataset aims to advance the study of clinical cognitive neuroscience, and contains highdensity task-based and task-free raw EEG data collected from TD individuals aged 6-44 years.

All participants were required to have sufficient EEG data (at least $24 \mathrm{~s}$ of artefact-free data) and for no measured EEG variables to fall $>3 \mathrm{SD}$ from the group mean (indicative of outlier activity). All participants meeting inclusion criteria were considered for matching. In total, 25 individuals from each pool were chronologically agematched to within 1 year, and sex-matched at a subgroup level split by age (16-25 years, 26-35 years, 36 years and over).

\section{EEG acquisition and pre-processing procedure}

Data from both groups was acquired using 128-channel EEG Geodesic Hydrocel nets (Electrical Geodesics, Inc., Eugene, OR, USA) with an appropriate size selected by measuring head circumference. In both datasets, electrode impedances were maintained below $50 \mathrm{k} \Omega$ during recording; the EEG signal was referenced to the vertex, and was recorded with a bandpass filter of 0.1 to 100
Hz. An amplifier gain of 10,000 was used for both datasets. Data from adults with DS was sampled at a rate of $250 \mathrm{~Hz}$, whilst TD control data was sampled at a rate of $500 \mathrm{~Hz}$.

During the resting-state task, participants of both groups repeated multiple eyes closed (EC) recording blocks. However, the first 11 participants with DS had one continuous 5.5 min EC block, which was then changed to multiple shorter blocks due to poor compliance and drowsiness (see Hamburg et al. [19] for details). All preprocessing was performed using EEGLAB [23] for MATLAB (MathWorks, Natick, MA) and was identical for both groups (see Hamburg et al. [19]). Briefly, the continuous EEG signal was digitally filtered using a lowpass filter of $30 \mathrm{~Hz}$. All data from six channels situated around the ears were removed due to poor fit (as a result of morphological differences in people with DS). Movement and blink artefacts were removed manually based on visual inspection. Bad channels were also identified based on visual inspection and were replaced using spherical spline interpolation. Remaining channels were re-referenced to the average electrode excluding VEOG and HEOG channels.

\section{EEG analysis}

Analysis was carried out using MATLAB (MathWorks, Natick, MA). For each individual, absolute and relative power measures for each frequency band of interest were obtained (delta $0.5-4 \mathrm{~Hz}$; theta 4-8 Hz; alpha 8-13 $\mathrm{Hz}$; beta 13-30 Hz) for each region (frontal and occipital; see Fig. 1 for electrode montages). Additionally, alpha peak features were calculated; this was defined as the frequency $(\mathrm{Hz})$ of the peak amplitude within the 8-13 $\mathrm{Hz}$ range.

Specifically, absolute power measures were obtained by convolving the raw signal from artefact free, nonoverlapping $2 \mathrm{~s}$ epochs for each channel with a five cycle Morlet wavelet. Power spectra were then averaged across all $2 \mathrm{~s}$ epochs, yielding a single average power spectrum for every electrode for each individual. Relative power measures were obtained for every electrode for each individual by dividing absolute power values by the total absolute power across the 0.1-30 Hz frequency range.

Some participants with DS did not have a measurable alpha peak. Standard methods would assign peak frequency to the lower boundary (i.e. $8 \mathrm{~Hz}$ ) for these individuals, as brain signals show a decrease in power with increasing frequency. Alpha peak features were therefore obtained for all individuals by removing the linear trend from individual power spectra to achieve 'spectral normalisation' [24]. This method allowed an accurate representation of these values to be obtained for all individuals, including those whose peak characteristics were initially lost within the natural EEG background. 


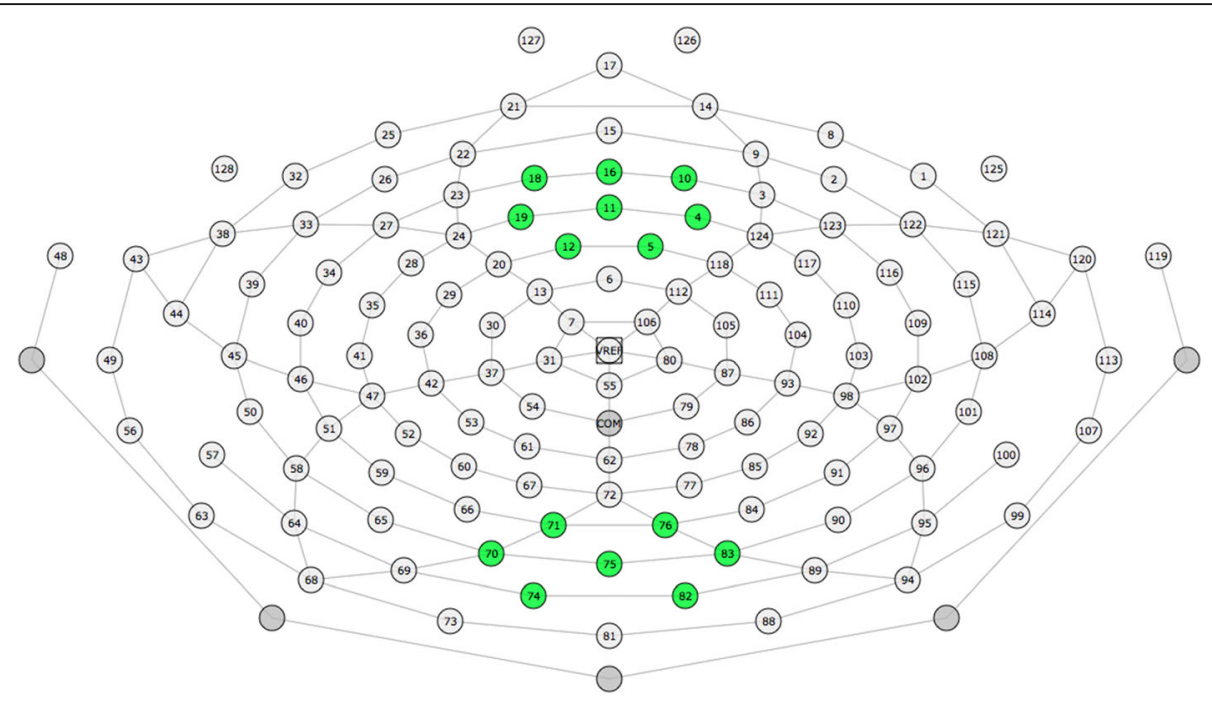

Fig. 1 Electrode map illustrating occipital and frontal montages. Electrode map illustrating individual cap electrodes for occipital (bottom cluster; $70(\mathrm{O} 1), 71,74,75(\mathrm{Oz}), 76,82,83(\mathrm{O} 2))$ and frontal (top cluster; 4 (F2), 5, 10, $11(\mathrm{Fz}), 12,16(\mathrm{AFz}), 18,19(\mathrm{~F} 1))$ montage averages used within this analysis. Corresponding 10-20 system shown in brackets where applicable

\section{Statistics and visualisation}

Customised MATLAB (MathWorks, Natick, MA) scripts were used to produce power-frequency spectrum plots. All statistical analyses were performed with SPSS.

In order to determine whether there was any effect of EC paradigm on EEG variables within individuals with DS (i.e. one segment of 5.5 minutes compared to 11 segments of 30 seconds), independent sample $t$ tests were used to compare absolute and relative power values between DS participants completing a full-block $(n=11)$ and those completing a split-block $(n=14)$ paradigm. As one $t$ test was significant (higher relative frontal theta power was found for the fullblock paradigm $(M=0.26(0.02 \mathrm{SD}))$ compared to the splitblock paradigm $(M=0.24$ (0.01 SD) $),(t(14.96)=2.35, p=$ $.033(95 \% \mathrm{CI}<0.01,0.02))$, EC paradigm was added as a covariate for all comparisons when activity was compared between groups.

All TD control participants were assigned to the splitblock protocol. ANCOVAs were used to statistically compare differences between groups. This was performed for each EEG variable at each region (occipital and frontal), using both absolute and relative power values and alpha peak amplitude, and alpha peak frequency values. Where the covariate (EC paradigm) was significant, this was left in the model, and where this was not significant, this was removed from the model. Partial eta squared values for each variable were used to provide an indication of effect size.

\section{Results}

\section{Preliminary analysis}

Final analyses were carried out on 25 individuals from each group. Table 1 shows the demographics of all participants included in the final analysis.
According to carer report of participants with DS, level of ID was mild $(n=13)$, moderate $(n=10)$, and severe $(n=2)$.

\section{EEG measures}

Table 2 shows absolute and relative values for each EEG measure by region within each group, in addition to statistical analysis of EEG variables by region. Standard deviations in the DS group appeared to be higher than those in the control group, particularly for peak frequency, indicative of more variability. Figure 2 (DS group) and Fig. 3 (TD control group) further illustrate increased variability within the DS group, apparent from individual power spectra. As a consequence, further analysis was undertaken to compare alpha peak frequency variance between groups, with highly significant between group effects found for both occipital $(F(24,24)=59.98, p<$ $.001)$ and frontal $(F(24,24)=29.15, p<.001)$ regions.

The overall group differences in the power-frequency spectra between DS and TD control participants are illustrated by Fig. 4 .

Statistical analysis of EEG variables from the occipital region revealed significantly higher absolute and relative delta power, and relative theta power, and significantly lower absolute and relative power in alpha and beta

Table 1 Participant demographics for each group

\begin{tabular}{lllll}
\hline Group & $\boldsymbol{n}$ & Mean age (SD) & Age range & Sex \\
\hline DS & 25 & $27.76(8.45)$ & $17-44$ & $12 \mathrm{M} ; 13 \mathrm{~F}$ \\
Control & 25 & $27.68(8.34)$ & $16-44$ & $14 \mathrm{M} ; 11 \mathrm{~F}$ \\
\hline
\end{tabular}

Participants were matched individually for age (years; within 1 year) and on a sub-group level for sex (sub-groups were age 16-25 years, 26-35 years, 36 years and over) 


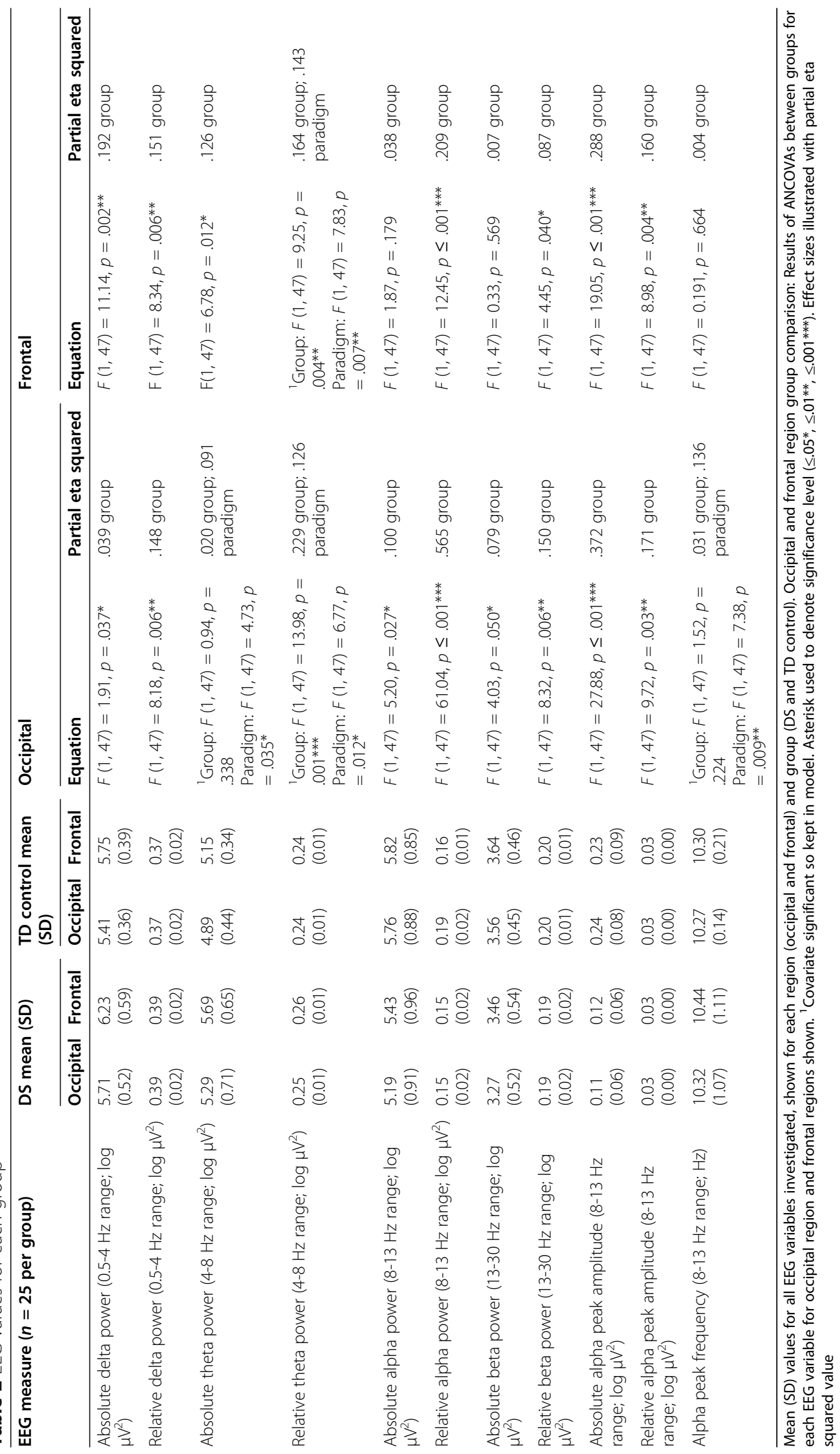



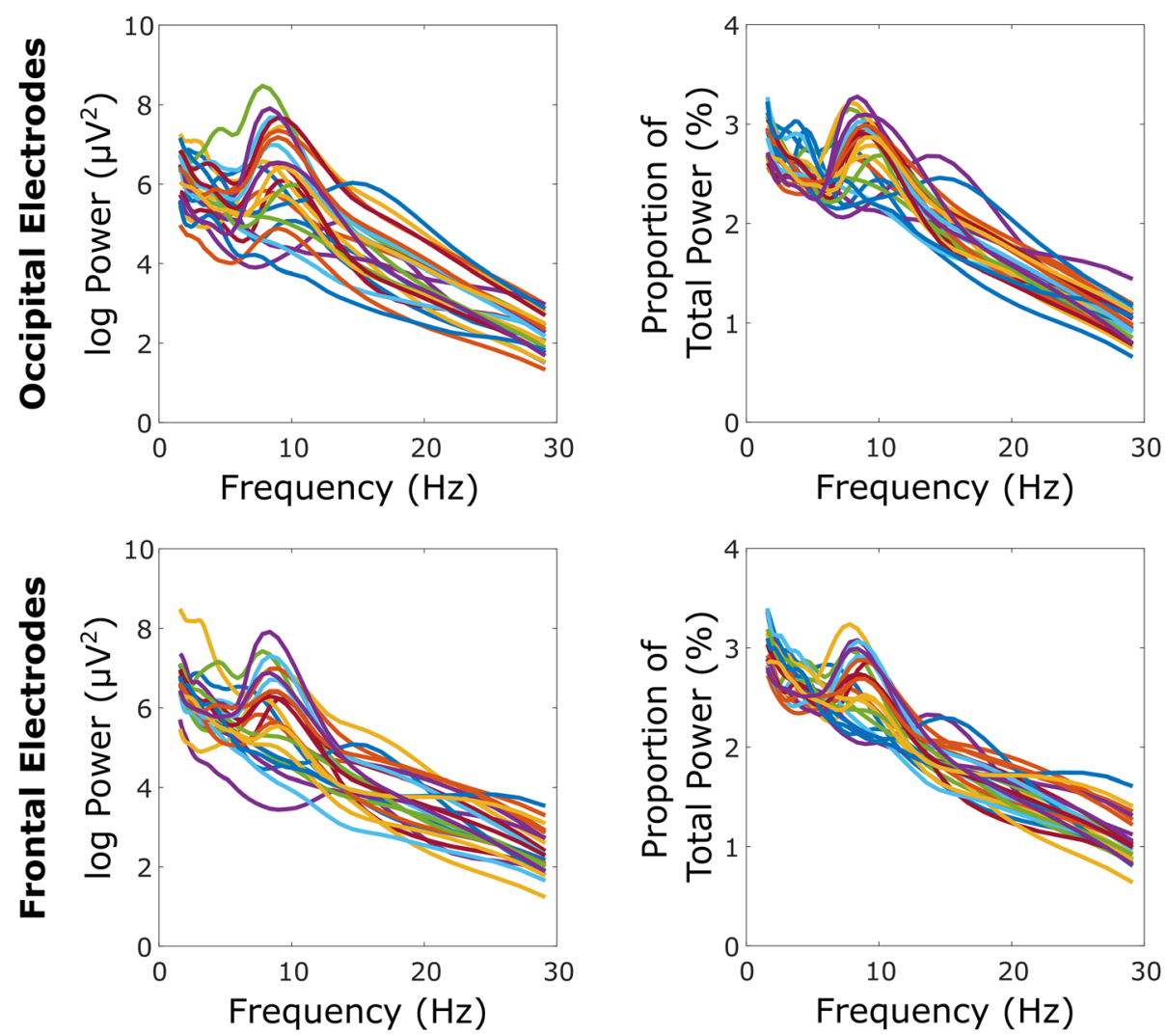

Fig. 2 DS power-frequency spectra. DS group power-frequency spectra for occipital (top) and frontal (bottom) regions. Absolute (left) and relative (right) values are shown for each individual

bands, for those with DS compared to TD controls (see Fig. 4 and Table 2). Those with DS also showed a significantly lower alpha peak amplitude. The effect sizes were greatest for relative alpha power, with group accounting for $56.5 \%$ of variance.

Results for the frontal region followed the same pattern (see Tables 2 and 3). All group differences (including both absolute and relative values) were statistically significant, apart from absolute alpha and beta power and alpha peak frequency. Overall, in this region, absolute and relative delta and theta power values were significantly higher in individuals with DS, whereas relative alpha and beta power values, and both absolute and relative alpha peak amplitude, were all significantly lower. The effect sizes were greatest for absolute alpha peak amplitude and relative alpha power, with group accounting for $28.8 \%$ and $20.9 \%$ of variance in these variables respectively.

It is worth noting that paradigm had a significant effect on occipital theta power, with both absolute and relative theta values higher for the full-block compared to the split-block paradigm for participants with DS (absolute values $5.57 \log \mu \mathrm{V}^{2}$ (0.63 SD) full-block and $5.08 \log \mu \mathrm{V}^{2}(0.71 \mathrm{SD})$ split-block; relative values 0.26 $\log \mu \mathrm{V}^{2}$ (0.02 SD) full-block and $0.25 \log \mu \mathrm{V}^{2}$ (0.01 SD) split-block). Paradigm also had a significant effect on relative theta power in the frontal region, with higher values for the full-block compared to the split-block paradigm $\left(0.26 \log \mu \mathrm{V}(0.02 \mathrm{SD})\right.$ full-block; $0.24 \log \mu \mathrm{V}^{2}$ (0.01 SD) split-block). Additionally, there was a significant relationship between alpha peak frequency and paradigm in the occipital region-those with DS completing the full-block paradigm had a faster peak (10.76 $\mathrm{Hz}(1.27 \mathrm{SD})$ ) compared with those completing the splitblock paradigm $(9.98 \mathrm{~Hz}(0.76 \mathrm{SD}))$. It is noteworthy that participants with DS completing the full-block also had higher standard deviation of occipital peak frequency (1.27 vs. 0.76), indicating more variability in this particular measure.

\section{Discussion}

This study aimed to characterise EEG differences between adults with genetically confirmed trisomy 21 with no evidence of dementia, and matched TD controls. We show an overall 'slower' EEG spectrum in both occipital and frontal regions (higher $0.5-8 \mathrm{~Hz}$ power and lower 8$30 \mathrm{~Hz}$ power) in people with DS. Alpha band activity in particular shows strong group differences, as shown by 

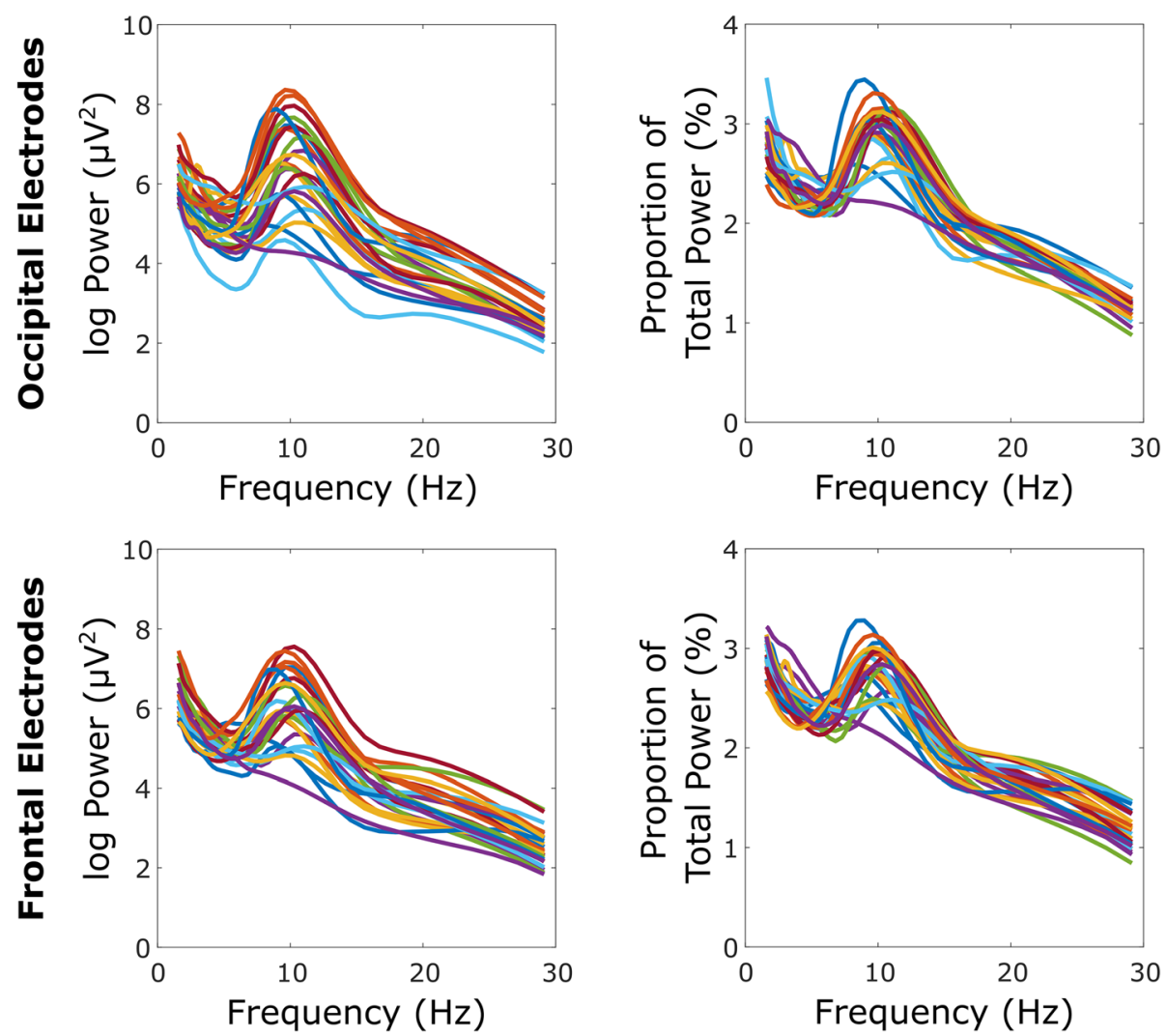

Fig. 3 TD power-frequency spectra. TD control group power-frequency spectra for occipital (top) and frontal (bottom) regions. Absolute (left) and relative (right) values are shown for each individual

the greatest effect sizes for group. We illustrate the value of using high-density EEG recordings to examine topographical differences and of utilising relative power measures in this population.

Higher delta activity [8-14], higher theta activity [6$14]$, lower alpha activity $[8,11,12]$, and lower beta activity $[12,13]$ have previously been reported in people with DS compared to TD controls. However, more evidence exists from previous research for higher than lower beta activity in people with DS [6-11, 14].

Interestingly, studies have linked 'slower' EEG spectra with cognitive impairment within the TD populationwith increased delta and decreased alpha associated with poor memory performance [25], and increased delta and theta, and decreased alpha, associated with mild cognitive impairment (MCI) [26, 27]. Such differences may also become more pronounced with progression from $\mathrm{MCI}$ to $\mathrm{AD}$ in the TD population [28, 29]. It is therefore possible that cognitive impairment has similar EEG signatures whether due to ID or neurodegenerative disease, characterised by a 'slower' spectra with more activity at lower frequencies. Furthermore, additional 'slowing' of the EEG spectra has also been linked to dementia in people with DS [30].
According to effect sizes, EEG characteristics most strongly associated with group were those related to alpha activity (occipital and frontal relative alpha power, and frontal peak amplitude). As discussed previously, differences in alpha band activity between individuals with DS and TD controls are commonly reported. Alpha peak frequency was not significantly associated with group in either region, which is in line with two previous studies $[10,13]$, though other studies have reported a slower peak frequency in individuals with DS $[6,7,11,14,20]$. The difference in peak frequency variability (as measured using the SD) in individuals with DS compared to TD controls is large $(1.07 \mathrm{~Hz}$ SD in DS; $0.14 \mathrm{~Hz}$ SD in TD controls), which may have impacted statistical power. Additionally, the highly significant group difference in alpha peak frequency variance suggests that peak frequency may be unstable in people with DS. This may be of particular importance as alpha peak frequency has been posited to act as an anchor around which the EEG spectrum is organised [17], and in turn the organisation of EEG activity represents the means by which neuronal networks dynamically communicate and interact [4]. 

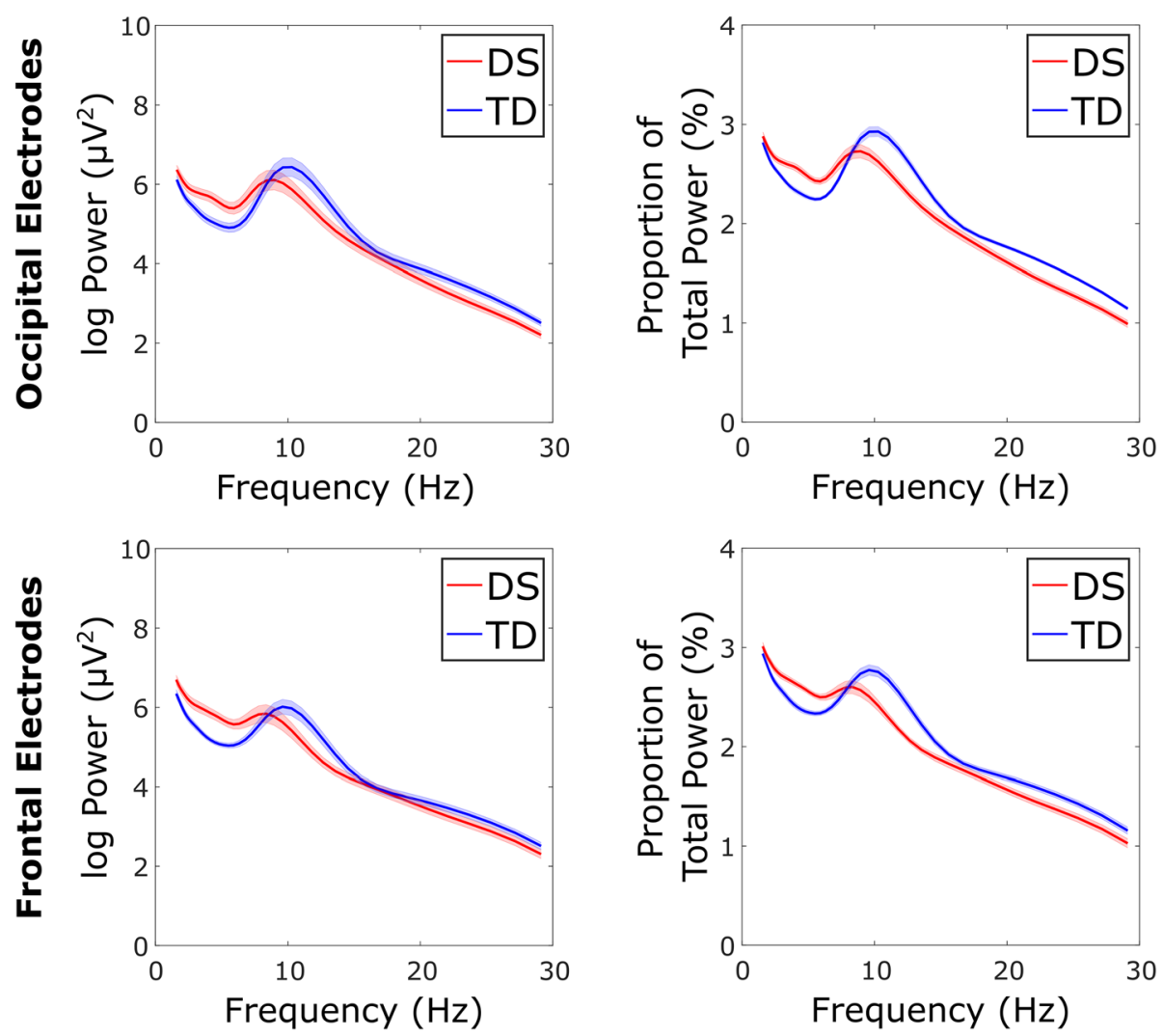

Fig. 4 Group comparison of power-frequency spectra. Comparison between DS group (red) and TD control group (blue) grand average powerfrequency spectra for occipital (top) and frontal (bottom) regions. Absolute (left) and relative (right) spectra are both shown

As all individuals with DS are expected to have AD neuropathology (amyloid plaques and tau tangles) by age 35 [31], the extent to which the group differences reported here are related to ID associated with the presence of an extra chromosome 21 and/or subclinical AD-neuropathology, remains unclear. Potential ID-related mechanisms include delayed and reduced brain maturation (with maturation in TD children associated with age-related reductions in delta and theta power, and age-related increases in alpha and beta power [32]) and over-inhibition [33-35]. Studies involving alternative populations of individuals with ID (e.g. fragile $\mathrm{X}$ ) would help elucidate whether these findings are unique to individuals with DS or are associated with ID in general. As the mean age of participants in this study is 27 years, which is prior to when significant amyloid-burden is expected in adults with DS [31], neuropathological mechanisms are unlikely. However, amyloid deposition can occur from childhood in DS [36] and therefore results may be confounded by this. Studies combining EEG with amyloid imaging (e.g. PET) could help explore this further. Future studies would also benefit from following individuals longitudinally, or examining different age groups cross-sectionally (including childhood and old age), to fully elucidate maturational and ageing influences.

For regional differences, stronger effect sizes were found for higher delta activity in adults with DS in frontal compared to occipital regions, which is in keeping with previous literature $[8,11,12]$. Frontally, differences in absolute values of alpha and beta power (although lower in DS) were not significant, yet reached significance occipitally, with greater effect sizes in occipital regions. Previous studies have also reported that differences in alpha and beta between people with DS and TD controls may be most apparent in posterior regions $[8,11]$. It is of note that participants with DS had larger SD values in frontal regions compared to occipital regions, which may have impacted statistical power here.

Effect sizes were generally larger for relative power values (possibly due to normalisation of relative values reducing variability and consequently increasing statistical power). Due to the high degree of variability in EEG measures of participants with DS, utilising relative values may be particularly beneficial in this population when comparing to TD subjects. Relative power helps account for differences in broadband power across participants, therefore, helping to control for 
Table 3 Summary of significant results

\begin{tabular}{|l|l|l|}
\hline & Occipital & Frontal \\
\hline $\begin{array}{l}\text { Delta power } \\
\text { (absolute) }\end{array}$ & & \\
\hline $\begin{array}{l}\text { Delta power } \\
\text { (relative) }\end{array}$ & & \\
\hline $\begin{array}{l}\text { Theta power } \\
\text { (absolute) }\end{array}$ & & \\
\hline $\begin{array}{l}\text { Theta power } \\
\text { (relative) }\end{array}$ & & \\
\hline $\begin{array}{l}\text { Alpha power } \\
\text { (absolute) }\end{array}$ & & \\
\hline $\begin{array}{l}\text { Alpha power } \\
\text { (relative) }\end{array}$ & & \\
\hline $\begin{array}{l}\text { Beta power } \\
\text { (absolute) }\end{array}$ & & \\
\hline $\begin{array}{l}\text { Beta power } \\
\text { (relative) }\end{array}$ & & \\
\hline $\begin{array}{l}\text { Peak amplitude } \\
\text { (absolute) }\end{array}$ & & \\
\hline $\begin{array}{l}\text { Peak amplitude } \\
\text { (relative) }\end{array}$ & & \\
\hline $\begin{array}{l}\text { Peak frequency } \\
\text { (Hz) }\end{array}$ & & \\
\hline $\begin{array}{l}\text { Peak frequency } \\
\text { variability }\end{array}$ & & \\
\hline
\end{tabular}

Light grey shows EEG measures that were significantly higher and dark grey shows measures that were significantly lower in participants with DS compared to TD controls

inter-individual variability in brain anatomy, which is particularly apparent in individuals with DS [37]. This anatomical variability may contribute to the higher EEG measure SD values found in this group. It is worth noting that absolute power values are likely to be of value when investigating individual differences between people with DS, as this variability is of interest.

The use of open source neuroimaging datasets is increasingly common and allows small exploratory studies of clinical population access to a large control group to obtain closely matched control subjects. Such datasets offer numerous benefits to researchers including increased efficiency, transparency and reproducibility [38]. Although variation in recording paradigm within the group with DS is a potential study limitation, effects were controlled for through the inclusion of this as a covariate. Furthermore, it appears likely that splitting the recording reduced participant drowsiness as intended (research suggests theta power is increased with light drowsiness [39] and theta power was higher for the fullblock paradigm). Serendipitously, this provides useful information pertaining to the most appropriate design for resting-state studies in people with DS.

This study benefitted from only including individuals with genetically confirmed trisomy 21 and the exclusion of individuals with cognitive decline (as assessed using the CAMDEX-DS) or a diagnosis of dementia. This is important as cognitive decline in DS has been associated with changes in EEG activity [30]. This ensured results were not influenced by any individuals with a rarer form of DS (for example mosaicism), and results are valid for individuals with DS prior to dementia onset. These variables are not commonly controlled for within DS studies, despite them substantially impacting the validity of findings. An additional strength of this study is that peak frequency measures were obtained by removing the individual linear trend from the EEG spectrum to achieve 'spectral normalisation'. This method has not been utilised in DS studies previously but is particularly useful in this population due to many individuals having a small peak that is not measurable beyond the natural background EEG noise.

A key limitation of this study is that there was no correction for multiple comparisons, due to the exploratory nature of this investigation-future studies should therefore prioritise replication of these findings. Future studies may also benefit from investigating differences in eyes-open EEG, and the examination of gamma activity in this population where possible. There is also an indication that the parietal region may be an area of particularly strong group differences (see Supplementary information), which future research may benefit from examining. Differences in inter-regional phase coupling between DS and TD groups also remains an important avenue for future investigation; however, there is a risk spurious increases in phase coupling may arise due to significant differences in oscillatory power between groups [40]. Such studies will therefore need to control for this.

Interestingly, we report here significantly lower alpha peak amplitude in individuals with DS. In line with this, previous research indicates that within individuals with DS, higher peak amplitude is associated with greater cognitive ability [19]. However, we also find here that theta power is significantly higher in individuals with DS compared to TD controls, despite previous research 
suggesting greater theta power may be associated with greater cognitive ability in individuals with DS [19]. It may therefore be the case that some EEG measures associated with higher cognitive ability in people with DS are closer to that of the TD population, whilst others are of the opposite direction (potentially suggestive of compensatory mechanisms). Importantly, therefore, higher ability individuals with DS may not necessarily have EEG activity closer to TD EEG spectra. The implications of this are interventions aiming to enhance cognitive ability in this population through seeking to 'normalise' EEG spectra could in fact negatively impact cognition. Instead, targeting EEG measures associated with individual differences in cognitive ability, rather than measures that differ between individuals with DS and TD controls, may be of benefit.

\section{Conclusions}

We report an overall 'slower' EEG spectrum, characterised by higher delta and theta power, and lower alpha and beta power, for frontal and occipital regions in people with DS. Alpha activity in particular shows strong group differences, including lower power, lower peak amplitude and greater peak frequency variance in people with DS. Such 'slowing' of the EEG spectrum has previously been associated with cognitive decline in both DS and TD populations. These findings indicate the potential existence of a universal EEG signature of cognitive impairment, regardless of origin (neurodevelopmental or neurodegenerative), warranting further exploration.

\section{Abbreviations}

AD: Alzheimer's disease; ANCOVA: Analysis of covariance; CAMDEXDS: Cambridge Examination of Mental Disorders of Older People with Down Syndrome and Others with Intellectual Disabilities; DS: Down syndrome; EEG: Electroencephalography; EC: Eyes closed; ID: Intellectual disability; MIPDB: Multimodal Resource for Studying Information Processing in the Developing Brain; SD: Standard deviation; TD: Typically developing

\section{Supplementary Information}

The online version contains supplementary material available at https://doi. org/10.1186/s11689-021-09392-z.

Additional file 1. Alpha power scalp plots. Scalp plots of alpha power contrasts for raw power group differences (left) and normalised power group differences (right).

\section{Acknowledgements}

The authors would like to thank all the participants and their carers, in addition to the Child Mind Institute for their open source dataset.

\section{Data availability}

EEG data analysed is available from the corresponding author upon reasonable request.

\section{Authors' contributions}

SH, CS and AS designed the study. SH and CS collected the data from participants with DS. SH and CS analysed the cognitive data. SH and DB analysed the EEG data. SH interpreted the results and wrote the manuscript. All authors read and approved the final manuscript.

\section{Funding}

This work was supported by the Baily Thomas Charitable Fund (TRUST/RNA/ AC/KW/3111/5776) and a Wellcome Trust Strategic Award (grant number: 098330/Z/12/Z) conferred upon The London Down Syndrome (LonDownS) Consortium

\section{Declarations}

\section{Ethics approval and consent to participate}

Ethical approval for the study was obtained from the North Wales West Research Ethics Committee (13/WA/0194). Where individuals had capacity to consent for themselves, written informed consent was obtained. Where individuals did not have capacity to consent for themselves, a consultee was asked to sign a form to indicate their decision regarding the individuals' inclusion based on their knowledge of the individual and his/her wishes, in accordance with the UK Mental Capacity Act 2005

\section{Consent for publication}

Not applicable.

\section{Competing interests}

The authors declare no competing interests.

\section{Author details}

${ }^{1}$ The London Down Syndrome Consortium (LonDownS), London, UK. ${ }^{2}$ Institute of Cognitive Neuroscience, University College London, London, UK. ${ }^{3}$ Queen Square Institute of Neurology, University College London, London, UK. ${ }^{4}$ Department of Forensic and Neurodevelopmental Sciences, Institute of Psychiatry, Psychology and Neuroscience, King's College London, London SE5 8AF, UK. 'Department of Psychology, University of Roehampton, London, UK.

Received: 3 May 2021 Accepted: 1 September 2021

Published online: 14 October 2021

\section{References}

1. de Graaf $G$, et al. Estimates of the live births, natural losses, and elective terminations with Down syndrome in the United States. Am J Med Genet A. 2015;167A(4):756-67. https://doi.org/10.1002/ajmg.a.37001.

2. Zis P, Strydom A. Clinical aspects and biomarkers of Alzheimer's disease in Down syndrome. Free Radic Biol Med. 2018;114:3-9. https://doi.org/10.1016/ j.freeradbiomed.2017.08.024.

3. Startin CM, et al. The LonDownS adult cognitive assessment to study cognitive abilities and decline in Down syndrome. Wellcome Open Res. 2016;1(11). https://doi.org/10.12688/wellcomeopenres.9961.1.

4. Buzsáki $G$, et al. Scaling brain size, keeping timing: evolutionary preservation of brain rhythms. Neuron. 2013:80(3):751-64. https://doi.org/10.1016/j. neuron.2013.10.002

5. Haegens $S$, et al. Inter- and intra-individual variability in alpha peak frequency. Neurolmage. 2014;92:46-55. https://doi.org/10.1016/j.neuroima ge.2014.01.049.

6. Ono Y. EEG changes with aging in adults with Down syndrome. Jpn J Psychiatry Neurol. 1993;47(1):75-84. https://doi.org/10.1111/j.1440-1819.1993. tb02033.x

7. Murata T, Koshino Y, Omori M, Murata I, Nishio M, Horie T, et al. Quantitative EEG study on premature aging in adult Down's syndrome. Biol Psychiatry. 1994;35(6):422-5. https://doi.org/10.1016/0006-3223(94)90010-8.

8. Medaglini S, et al. P300 and EEG mapping in Down's syndrome. J Psychophysiol. 1997;11(1):48-58 https://moh-it.pure.elsevier.com/en/publica tions/p300-and-eeg-mapping-in-downs-syndrome.

9. Partanen J, Soininen H, Könönen M, Kilpeläinen R, Helkala EL, Riekkinen P Sr. EEG reactivity correlates with neuropsychological test scores in Down's syndrome. Acta Neurol Scand. 1996;94(4):242-6. https://doi.org/10.1111/j.1 600-0404.1996.tb07059.x.

10. Politoff $A L$, et al. Cognition-related EEG abnormalities in nondemented Down syndrome subjects. Dementia (Basel, Switzerland). 1996;7(2):69-75. https://doi.org/10.1159/000106856. 
11. Locatelli T, et al. Quantified electroencephalogram in adult patients with Down's syndrome. Dev Brain Dysfunct. 1996;9(4):215-23.

12. Babiloni $C$, Albertini $G$, Onorati $P$, Vecchio $F$, et al. Inter-hemispheric functional coupling of eyes-closed resting EEG rhythms in adolescents with Down syndrome. Clin Neurophysiol. 2009;120(9):1619-27. https://doi.org/1 0.1016/j.clinph.2009.06.017.

13. Babiloni C, Albertini G, Onorati P, Muratori C, et al. Cortical sources of EEG rhythms are abnormal in Down syndrome. Clin Neurophysiol. 2010;121(8): 1205-12. https://doi.org/10.1016/j.clinph.2010.02.155.

14. Velikova $\mathrm{S}$, et al. Cognitive impairment and EEG background activity in adults with Down's syndrome: a topographic study. Hum Brain Mapp. 2011; 32(5):716-29. https://doi.org/10.1002/hbm.21061.

15. Kreezer G. Intelligence level and the occipital alpha rhythm in the Mongolian type of mental deficiency. Am J Psychol. 1939;52(4):503-32. https://doi.org/10.2307/1416467.

16. Anokhin A, Vogel F. EEG alpha rhythm frequency and intelligence in normal adults. Intelligence. 1996;23(1):1-14. https://doi.org/10.1016/S0160-2 896(96)80002-X.

17. Klimesch W. EEG alpha and theta oscillations reflect cognitive and memory performance: a review and analysis. Brain Res Brain Res Rev. Apr. 1999;29(23):169-95. https://doi.org/10.1016/s0165-0173(98)00056-3.

18. Clark R, et al. Spontaneous alpha peak frequency predicts working memory performance across the age span. Int J Psychophysiol. 2004;53(1):1.

19. Hamburg S, et al. Dynamic causal modeling of the relationship between cognition and theta-alpha oscillations in adults with Down syndrome. Cerebral Cortex (New York, NY). 2019;29(5):2279-90. https://doi.org/10.1093/ cercor/bhz043.

20. Soininen H, Partanen J, Jousma“ki V, Helkala EL, Vanhanen M, Majuri S, et al. Age-related cognitive decline and electroencephalogram slowing in Down's syndrome as a model of Alzheimer's disease. Neuroscience. Mar. 1993;53(1): 57-63. https://doi.org/10.1016/0306-4522(93)90284-m.

21. Ball SL, Holland AJ, Huppert FA, Treppner P, Watson P, Hon J. The modified CAMDEX informant interview is a valid and reliable tool for use in the diagnosis of dementia in adults with Down's syndrome. J Intellect Disabil Res. Sept. 2004;48(Pt 6):611-20. https://doi.org/10.1111/j.1365-2788.2004. 00630.x

22. Langer $\mathrm{N}$, et al. A resource for assessing information processing in the developing brain using EEG and eye tracking. Scientific Data. 2017:4:170040.

23. Delorme A, Makeig S. EEGLAB: an open source toolbox for analysis of single-trial EEG dynamics including independent component analysis. J Neurosci Methods. Mar. 2004;134(1):9-21. https://doi.org/10.1016/j. jneumeth.2003.10.009.

24. Demanuele $C$, et al. Distinguishing low frequency oscillations within the $1 / f$ spectral behaviour of electromagnetic brain signals. Behav Brain Funct. 2007;3:62. https://doi.org/10.1186/1744-9081-3-62.

25. Babiloni C, Cassetta E, Binetti G, Tombini M, del Percio C, Ferreri F, et al. Resting EEG sources correlate with attentional span in mild cognitive impairment and Alzheimer's disease. Eur J Neurosci. 2007;25(12):3742-57. https://doi.org/10.1111/j.1460-9568.2007.05601.x

26. Guner D, et al. Contribution of quantitative EEG to the diagnosis of early cognitive impairment in patients with idiopathic Parkinson's disease. Clin EEG Neurosci. 2017:48(5):348-54. https://doi.org/10.1177/1550059416662412.

27. Gouw AA, et al. EEG spectral analysis as a putative early prognostic biomarker in nondemented, amyloid positive subjects. Neurobiol Aging. 2017:57:133-42. https://doi.org/10.1016/j.neurobiolaging.2017.05.017.

28. Hsiao F-J, et al. Altered oscillation and synchronization of default-mode network activity in mild Alzheimer's disease compared to mild cognitive impairment: an electrophysiological study. PLoS One. 2013;8(7):e68792. https://doi.org/10.1371/journal.pone.0068792.

29. Scrascia, Federica, et al. "Relationship among diffusion tensor imaging, EEG activity, and cognitive status in mild cognitive impairment and Alzheimer's disease patients." J Alzheimers Dis, vol. 38, no. 4, 2014, pp. 939-50, doi: https://doi.org/10.3233/JAD-130788

30. Musaeus CS, Salem LC, Sabers A, Kjaer TW, Waldemar G. Associations between electroencephalography power and Alzheimer's disease in persons with Down syndrome. J Intellect Disabil Res. 2019;63(9):1151-7. https://doi. org/10.1111/jir.12627.

31. Mann DM. Alzheimer's disease and Down's syndrome. Histopathology. Aug. 1988;13(2):125-37. https://doi.org/10.1111/j.1365-2559.1988.tb02018.x.
32. Cragg L, Kovacevic N, McIntosh AR, Poulsen C, Martinu K, Leonard G, et al. Maturation of EEG power spectra in early adolescence: a longitudinal study. Dev Sci. 2011;14(5):935-43. https://doi.org/10.1111/j.1467-7687.2010.01031.x.

33. Fernandez F, Morishita W, Zuniga E, Nguyen J, Blank M, Malenka RC, et al. Pharmacotherapy for cognitive impairment in a mouse model of Down syndrome. Nat Neurosci. 2007;10(4):411-3. https://doi.org/10.1038/nn1860.

34. Braudeau J, Delatour B, Duchon A, Pereira PL, Dauphinot $L$, de Chaumont F, et al. Specific targeting if the GABA-A receptor A5 subtype by a selective inverse agonist restores cognitive deficits in Down syndrome mice. J Psychopharmacol. 2011;25(8):1030-42. https://doi.org/10.1177/02698811114 05366.

35. Cotestabile A, et al. The GABAergic hypothesis for cognitive disabilities in Down syndrome. Front Cell Neurosci. 2017;11:54.

36. Lemere CA, Blusztajn JK, Yamaguchi H, Wisniewski T, Saido TC, Selkoe DJ. Sequence of deposition of heterogeneous amyloid beta-peptides and APO E in Down syndrome: implications for initial events in amyloid plaque formation. Neurobiol Dis. Feb. 1996;3(1):16-32. https://doi.org/10.1006/ nbdi.1996.0003

37. Lee $\mathrm{N}$, et al. Dissociations in cortical morphometry in youth with Down syndrome: evidence for reduced surface area but increased thickness. Cereb Cortex. 2016;26(7):2982-90. https://doi.org/10.1093/cercor/bhv107.

38. Gilmore L, Cuskelly M. Associations of child and adolescent mastery motivation and self-regulation with adult outcomes: a longitudinal study of individuals with Down syndrome. Am J Intellect Dev Disabil. 2017;122(3): 235-46. https://doi.org/10.1352/1944-7558-122.3.235.

39. Britton, J, et al. Electroencephalography (EEG): an introductory text and atlas of normal and abnormal findings in adults, children and infants. Chicago: American Epilepsy Society; 2016.

40. Muthukumaraswamy SD, Singh KD. A cautionary note on the interpretation of phase-locking estimates with concurrent changes in power. Clin Neurophysiol. 2011;122(11):2324-5. https://doi.org/10.1016/j.clinph.2011.04. 003.

\section{Publisher's Note}

Springer Nature remains neutral with regard to jurisdictional claims in published maps and institutional affiliations.

Ready to submit your research? Choose BMC and benefit from:

- fast, convenient online submission

- thorough peer review by experienced researchers in your field

- rapid publication on acceptance

- support for research data, including large and complex data types

- gold Open Access which fosters wider collaboration and increased citations

- maximum visibility for your research: over $100 \mathrm{M}$ website views per year

At $\mathrm{BMC}$, research is always in progress.

Learn more biomedcentral.com/submissions 